\title{
Profile of Weibo Song
}

Dr. Weibo Song received his Ph.D. degree in the Zoological Institute, Bonn University, Germany, in January 1989. He then joined the College of Fisheries, Ocean University of China (OUC), and was qualified as a lecturer, associate professor and full professor in the following 4 years. During the past two decades, Dr. Song established the Laboratory of Protozoology and led several researchers working on taxonomy, cell development (pattern formation of cortical structure), systematics and ecology of ciliated protozoa mainly from marine biotopes in Bohai Bay and Yellow Sea. In addition, a survey on pathogenic ciliates in mariculture had been performed over a 10-year-peirod (1993-2003). Since 2005 , Dr. Song's group began to carry out the fauna projects in tropical and subtropical area mainly along Guangdong coasts, focusing on mangrove, estuary and brackish waters, off-shore habitats and all kinds of sea-shore wetlands. Meanwhile, many patterns in cell division have been revealed for the first time, especially in hypotrichs and other higher taxa. Since 1997, Dr. Song and his colleagues/students incorporated molecular methods into their studies, regarding species identification, systematic relationships and phylogenetic evolution. Covering the fields mentioned above, Dr. Song, together with his team members, published three monographs and over 270 papers in international peer-reviewed journals.

In 1994, Dr. Song received the National Science Fund for Distinguished Young Scholars from the National Natural Science Foundation of China, and was appointed as the professor of the Changjiang Scholarship Program in OUC by the Ministry of Education in 1999. And Dr. Song is honored as the Chinese Young Scientists (2005). Dr. Song is currently the Chief of the Institute of Evolution \& Marine Biodiversity, OUC, the Executive Member of the International Society of Protistologists, the Chairman of Asian Congress of Protistology and the President of the Chinese Society of Protozoology. In 2015, Dr. Song was elected as an Academician of Chinese Academy of Sciences.

\section{Diversity and taxonomy of marine ciliates}

Ecosystem function in aquatic habitats is largely controlled

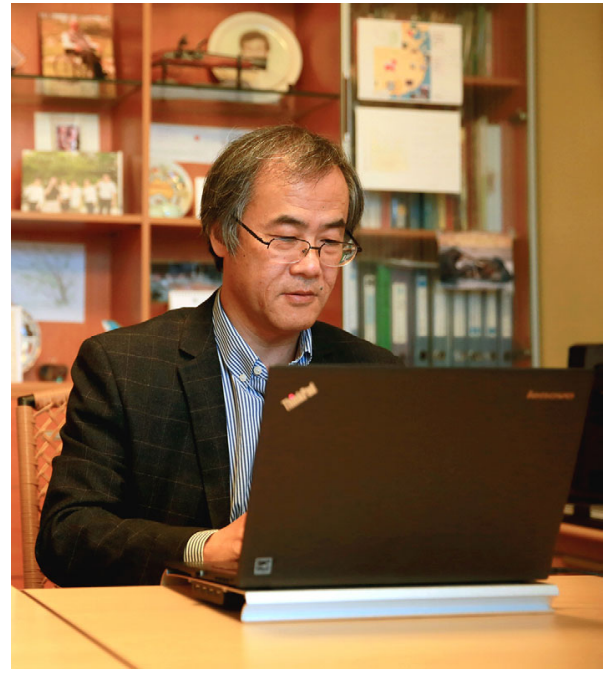

by microbial processes. Microeukaryotes in general, and ciliates in particular, serve as a number of critical ecological roles including: (i) influencing the control of the carbon cycle; (ii) providing a food source for juvenile fish and shellfish; (ii) some are important pathogens of aquatic animals; (iv) many can be used as indicators of water quality and ecosystem function. Nevertheless, knowledge of microeukaryotes is scant, and the diversity and fauna of marine ciliated protozoa are largely unknown, especially in East Asia or western Pacific Ocean area.

Since later 1980s, Dr. Song's group has dedicated themselves into the fauna, ecological function, community structure and geographic distribution of free-living ciliates in north and south China seas, and presented a large series of new data. In addition, a general survey of pathogenic protozoa in cultured aquatic animals was also carried out during the period of 1993-2003. The results include: (i) a 25-year taxonomic survey of ca. 20 orders of un- or poorly-known marine forms in north China seas with the description of over 200 new species and many higher taxa (new genera, families and orders etc.). The main work was summarized in the monograph "Free-living ciliates in the Bohai and Yellow Sea, China" in 2009. (ii) as the first 
monograph about pathogenic/parasitic protozoa isolated from mollusks, shrimps and fishes in mariculture waters, a book entitled "Pathogenic Protozoa in Mariculture" was published, in which over 200 species, including many new, of ciliates and myxosporans have been reported. (iii) investigations on tropical ciliate fauna. Mangroves serve as a number of important functions including protecting coasts from erosion and surge storms, attenuating marine pollution, controlling carbon fluxes, providing habitats for commercially important fish and shellfish, and supporting a wide variety of organisms thus enhancing biodiversity. Since 2005, Dr. Song's group has conducted surveys on ciliates in Guangdong costal regions, and obtained many first-hand data that greatly contribute to the ecological and environmental biology. (iv) conservation of Jiaozhou Bay-biodiversity assessment and bio-monitoring using ciliates. Jiaozhou Bay is a major center for fisheries and marine industries, but water quality is deteriorating as a result of pollution. Together with his colleagues in the Natural History Museum, UK, Dr. Song's group acquired a 5-year joint project supported by National Natural Science Foundation of China (NSFC) and Darwin Initiative Programme, UK. The project developed a bio-monitoring indicator tool, enhancing professional skills relating to ciliate biodiversity and water quality assessment, and developing a guide for identification of ciliates.

\section{Ontogenetic studies on ciliates}

Ciliates, especially hypotrichs and euplotids, exhibit a great diversity in cortical development and pattern formation during cell division, which documents tremendous information in studies of evolution and systematics, as well as genetics and cell biology. Supported by several NSFC projects, Dr. Song and his group have completed ontogenetic studies on representatives of over 60 genera and revealed numerous important novel patterns and phenomena, which significantly promote the development of ontogenetic investigations. For this work, Dr. Song won the First Class Natural Science Award issued by the Chinese Ministry of Education.

Three most representative achievements are as follows: (1) details of the morphogenetic process in the genus Kiitricha, a well-known ancestor of hypotrichs, was described for the first time, and the results show an extremely unusual developmental pattern compared with all other known forms in the class Spirotrichea. (2) discocephalines are cephalized organisms that are traditionally considered to be hypotrichs $(s . l$. ) but their precise systematic position has long been uncertain. These organisms also exhibit several intermediate morphological and morphogenetic features, and hitherto none have been investigated using molecular methods. Using the species, Prodiscocephalus borrori, the cortical development was observed during binary division and several important points have been revealed: (i) in the parental AZM, only the posterior end is renewed by dedifferentiation of the old structures; (ii) the oral primordium in the opisthe occurs de novo on the cell surface as seen in other typical stichotrichs; (iii) in both dividers, the UManlage does not split longitudinally in the usual way, but instead divides transversely to form the paroral and endoral membranes. Thus discocephalines exhibit more similarities to stichotrichs than to euplotids and represent an intermediate group between the euplotids and the StichotrichiaOligotrichia-Choreotrichia assemblage. (3) morphogenesis during the binary fission of a stichotrich ciliate Pseudoamphisiella alveolata was investigated. The process is characterized as follows: (i) the oral primordium in the opisthe and the FVT-anlagen in both dividers are formed de novo on the cell surface; (ii) an "extra" anlage, which is generated on the right of the right marginal anlage, develops into 3 or 4 "extra" marginal cirri that connect the caudal cirri with the marginal rows; (iii) the right marginal anlage is formed within the old structure, and unlike most stichotrichs, the right marginal anlagen in both dividers generate closely together.

\section{Phylogenetic studies on ciliates}

Phylogenetic analysis has been rapidly emerging as a popular approach to reveal the evolution history and diversity of species in the latter half of the 20th Century. However, the lack of gene marker sequencing of ciliates posed an enormous obstacle to the widely application of phylogenetic analyses in this field. Since the end of last century, Dr. Song and his team have established a DNA library including a wide range of ciliates from temperate and tropical waters of China, and sequenced their phylogenetic markers, for example, the ribosomal RNA (rRNA) gene. On this basis, Dr. Song' group clarified the positions of many important taxa in evolution and taxonomy, and became one of the internationally top ranked teams in this field. According to the recent statistics of Web of Science, Dr. Song' group and his collaborators have published 129 phylogenetic related papers on peer-reviewed journals in the past ten years, accounting for $14 \%$ of publications (916 in total) in their field.

Till now, Dr. Song and his team have carried out phylogenetic studies in many important taxa, such as hypotrichs, euplotes, oligotrichs, scuticociliatids, cyrtophorids etc. The achievements can be summarized as follows: (i) establishment of the largest DNA library of marine ciliates in the world, covering more than 1,700 species/populations and over a half of orders (32/59) in the phylum Ciliophora, and contributed more than $50 \%$ of DNA sequencing data among the key taxa, like hypotrichs, in the GenBank database. (ii) based on multi-gene analysis, evolutionary and taxonomic positions of over 50 orders in the phylum Ciliophora have been reviewed and clarified. (iii) establishment of three novel classes, six new orders/suborders after clarifying the intra-relationships within the class Spirotrichea. (iv) several 
suggestions/hypotheses have been proposed to improve topologies of ciliated phylogenetic trees, such as including unidentified environmental sequences, and taking secondary structures of rRNA genes into account. These works were published in mainstream journals such as Molecular Phylogenetics and Evolution, Proceedings of the Royal Society of London B, etc. Their work enriched our knowledge of ciliate evolution, and laid a solid foundation for future investigations such as how to select phylogenetic markers.

\section{DNA barcoding of ciliates}

Scientists found that the undiscovered species diversity among ciliates may be magnitude greater than previously thought, as molecular techniques, i.e. DNA fingerprinting, cloning library, high-throughput sequencing, were performed in ecological study. DNA barcoding provides a reliable and rapid method for species identification, and compensates for the lack of diagnostic morphological features within ciliates. Investigations on DNA barcoding of ciliates are still in the very early stage, though nowadays numbers of unidentified OTUs (operational taxonomic units) are exploding in molecular ecological study.

The achievements can be summarized as two points: (i) several mitochondrial and nuclear candidate barcode markers had been used in DNA barcoding of ciliates in previous studies, but no one was widely accepted. Research group led by Dr. Song tested the ability of eight candidate barcode markers, cytochrome $c$ oxidase subunit I gene $(C O I), \mathrm{V} 2$ and V4 region of 18SrRNA, D1 and D2 region of 28SrRNA, internal transcribed spacer 1, 2 (ITS1, ITS2) and 5.8SrRNA, to identify ciliate species. The work revealed that among these eight candidate markers, COI gene, ITS1, ITS2, and D2 region of 28SrRNA gene are reliable for species identification. By contrast, no barcoding gap is detected in V2 and V4 region of 18SrRNA gene, D1 region of 28SrRNA gene, and 5.8SrRNA gene. In addition, Dr. Song's group firstly reported mitochondrial heteroplasmy of ciliates, which revealed genetic differences of mitochondrial COI gene within and among individual cells and populations. (ii) extensive sampling and accurate identification are constraints of construction of an integrated database, which is the final task of DNA barcoding. Based on the largest DNA library of marine ciliates in the world, which were accurately identified by experienced taxonomists, barcoding sequences as well as morphological and other characters of 83 ciliated species have been obtained by Dr. Song's group. This primary DNA barcoding database includes species of Oligohymenophorea and Spirotrichea and database improving is still on-going to incorporate more species.

Open Access This article is distributed under the terms of the Creative Commons Attribution License which permits any use, distribution, and reproduction in any medium, provided the original author(s) and source are credited. 